\title{
The pharmacodynamic effect of terlipressin versus high-dose octreotide in reducing hepatic venous pressure gradient: a randomized controlled trial
}

\author{
Bing Li ${ }^{1 \#}$, Jie Chen ${ }^{2,3 \#}$, Chun-Qing Zhang ${ }^{4} \wedge$, Guang-Chuan Wang ${ }^{4} \wedge$, Jin-Hua Hu ${ }^{4} \wedge$, Jian-Jun Luo ${ }^{5}$, \\ Wen Zhang ${ }^{6}$, Yi-Chao Wei ${ }^{6}$, Xiao-Qing Zeng ${ }^{2,3}$, Shi-Yao Chen ${ }^{2,3,7}$ \\ ${ }^{1}$ Department of Infectious Diseases, Zhongshan Hospital, Fudan University, Shanghai, China; ${ }^{2}$ Department of Gastroenterology and Hepatology, \\ Zhongshan Hospital, Fudan University, Shanghai, China; ${ }^{3}$ Center of Evidence-Based Medicine, Fudan University, Shanghai, China; ${ }^{4}$ Department \\ of Gastroenterology, Shandong Provincial Hospital Affiliated to Shandong University, Jinan, China; ${ }^{5}$ Department of Interventional Radiotherapy, \\ Zhongshan Hospital Fudan University, Shanghai, China; ${ }^{6}$ Department of Geratology, Zhongshan Hospital Fudan University, Shanghai, China; \\ ${ }^{7}$ Endoscopy Center and Endoscopy Research Institute, Zhongshan Hospital, Fudan University, Shanghai, China \\ Contributions: (I) Conception and design: B Li, J Chen; (II) Administrative support: SY Chen; (III) Provision of study materials or patients: B Li, J \\ Chen; (IV) Collection and assembly of data: B Li, J Chen, CQ Zhang, JJ Luo, W Zhang, GC Wang; (V) Data analysis and interpretation: All authors; \\ (VI) Manuscript writing: All authors; (VII) Final approval of manuscript: All authors. \\ \#These authors contributed equally to this work. \\ Correspondence to: Shi-Yao Chen. Department of Gastroenterology and Hepatology, Zhongshan Hospital, Fudan University, Shanghai, China; Center \\ of Evidence-Based Medicine, Fudan University, Shanghai, China; Shanghai Institute of Liver Disease, Shanghai, China; Endoscopy Center and \\ Endoscopy Research Institute, Zhongshan Hospital, Fudan University, Shanghai, China. Email: chen.shiyao@zs-hospital.sh.cn.
}

Background: Vasoactive drugs can reduce portal venous pressure and control variceal bleeding. However, few studies have explored the hemodynamic effects of terlipressin and high-dose octreotide in such patients. Our purpose was to evaluate the hemodynamic changes and safety of using terlipressin and high-dose octreotide in patients with decompensated liver cirrhosis.

Methods: A multi-center randomized controlled trial was conducted. Cirrhotic patients with a history of variceal bleeding were included. Terlipressin or high-dose octreotide was administered during the procedure of measuring hepatic venous pressure gradient (HVPG). Hemodynamic parameters and symptoms were recorded.

Results: A total of 88 patients were included. HVPG was significantly reduced at 10, 20, and 30 min after drug administration in the terlipressin group $(16.3 \pm 6.4$ vs. 14.7 $\pm 5.9,14.0 \pm 6.1$, and 13.8 \pm 6.1 , respectively, $\mathrm{P}<0.001)$ and the high-dose octreotide group $(17.4 \pm 6.6$ vs. $15.1 \pm 5.8,15.3 \pm 6.2$, and $16.1 \pm 6.0$, respectively $\mathrm{P}<0.01)$. Decreased heart rate and increased mean arterial pressure were more often observed in the terlipressin group. The overall response rates were not significantly different between the groups (52.8\% vs. $44.8 \%, \mathrm{P}=0.524)$. The terlipressin group had significantly higher response rates at $30 \mathrm{~min}$ compared to the high-dose octreotide group in those with alcoholic liver cirrhosis [6/6 (100\%) vs. 0/4 (0\%), $\mathrm{P}=0.005]$. The incidence of adverse drug events was rare and similar in the two groups.

Conclusions: Both terlipressin and high-dose octreotide were effective and safe for reducing HVPG. The pharmacodynamic effect of terlipressin persisted longer. The terlipressin group had higher response rates in those with alcoholic cirrhosis (trial number: NCT02119884).

Keywords: Portal hypertension; liver cirrhosis; terlipressin; octreotide

\footnotetext{
^ ORCID: Bing Li, 0000-0002-8727-3591; Jie Chen, 0000-0002-8295-6350; Chun-Qing Zhang, 0000-0001-8711-1579; Guang-Chuan Wang, 0000-0003-1228-1084; Jin-Hua Hu, 0000-0003-2541-6697; Jian-Jun Luo, 0000-0003-4942-0439; Wen Zhang, 0000-0002-2301147X; Yi-Chao Wei, 0000-0002-1469-3341; Xiao-Qing Zeng, 0000-0003-3494-8636; Shi-Yao Chen, 0000-0002-0873-9198.
} 
Submitted Oct 07, 2020. Accepted for publication Jan 31, 2021.

doi: 10.21037/atm-20-6774

View this article at: http://dx.doi.org/10.21037/atm-20-6774

\section{Introduction}

The Baveno VI consensus (1) concluded that vasoactive drugs are first-line medicines for treating acute variceal bleeding, even before emergency endoscopy. A previous meta-analysis showed that octreotide, an analog of somatostatin, had higher hemostasis rates than other traditional vasoactive drugs (2). However, more recent studies failed to demonstrate a difference in efficacy among somatostatin, octreotide, and terlipressin (3-5). Thus, there is still controversy over the pharmacodynamic effects of these vasoactive drugs.

The main mechanism by which vasoactive drugs control variceal hemorrhage is through reducing portal venous pressure. Hepatic venous pressure gradient (HVPG) is the gold standard for estimating portal venous pressure (6). HVPG predicts poor response and prognosis after acute variceal bleeding (1). A systemic review demonstrated that a reduction in HVPG below $12 \mathrm{mmHg}$ or by more than $20 \%$ from baseline could effectively prevent variceal bleeding (7-9). A study also revealed that terlipressin's hemodynamic effect was more prolonged than regular-dose octreotide (10). Since high-dose somatostatin could achieve more persistent hemodynamic effects than the regular dose (11), there is a need to investigate the true effects of high-dose octreotide.

The purpose of this study was to evaluate the hemodynamic changes and safety of using terlipressin and high-dose octreotide in patients with decompensated liver cirrhosis. We present the following article in accordance with the CONSORT reporting checklist (available at http:// dx.doi.org/10.21037/atm-20-6774).

\section{Methods}

This study was a randomized open-label controlled clinical trial with 2 centers in China. Cirrhotic patients with variceal bleeding history who underwent HVPG measurements in Zhongshan Hospital and Shandong Provincial Hospital between February 1, 2014, and January 31, 2016, were included. The diagnosis of cirrhosis was based on liver biopsy or imaging results. Gastroesophageal varices were confirmed by endoscopy. The exclusion criteria included: (I) $<18$ years old or $>72$ years old; (II) history of splenectomy or transjugular intrahepatic portosystemic shunt (TIPS); (III) the presence of severe complications, including spontaneous bacterial peritonitis, hepatorenal syndrome, or uncontrolled hepatic encephalopathy; (IV) the presence of portal or hepatic vein thrombosis; (V) pregnant or lactating women; (VI) contraindications to terlipressin or octreotide such as ischemic cardiovascular disease; (VII) under pharmacotherapy of non-selective $\beta$-blockers; (VIII) refusal to participate.

The study was conducted following the Declaration of Helsinki (as revised in 2013). The ethics board approved the study of Zhongshan Hospital and Shandong Provincial Hospital (No. B2014-025). The study was registered on http://www.clinicaltrials.gov (NCT02119884). Written consent was obtained from each patient involved in the study.

\section{HVPG measurements}

HVPG measurements were performed after an overnight fast under monitoring and local anesthesia. A 7F catheter sheath (Johnson \& Johnson, New Brunswick, NJ, USA) was placed in the right internal jugular vein after the puncture. A 4F Cobra catheter and a VER guide wire (402-607x; Johnson \& Johnson) were inserted through the catheter sheath into the inferior vena cava. A stiff guidewire (RF*PA35263M; Terumo, Tokyo, Japan) was then exchanged in the catheter, and a balloon catheter (Synergy, Boston, MA, USA) was inserted into the hepatic vein. The balloon (diameter: $10 \mathrm{~mm}$, length: $4 \mathrm{~cm}$ ) was placed in the hepatic vein, $2 \mathrm{~cm}$ next to the opening of the inferior vena cava. A pressure transducer (42584; ICU Medical Inc., San Clemente, CA, USA) was then connected. Free hepatic venous pressure (FHVP) was measured after zero calibration. When the balloon was inflated in the hepatic vein, a contrast medium was injected to check whether a typical wedged pattern was formed without communication. If complete occlusion was confirmed, wedged hepatic venous pressure (WHVP) was measured after repeated zero calibration. Each WHVP and FHVP measurement was acquired 3 times to determine the average value. HVPG was defined as the difference between WHVP and FHVP. After obtaining the baseline HVPG value, vasoactive drugs were 


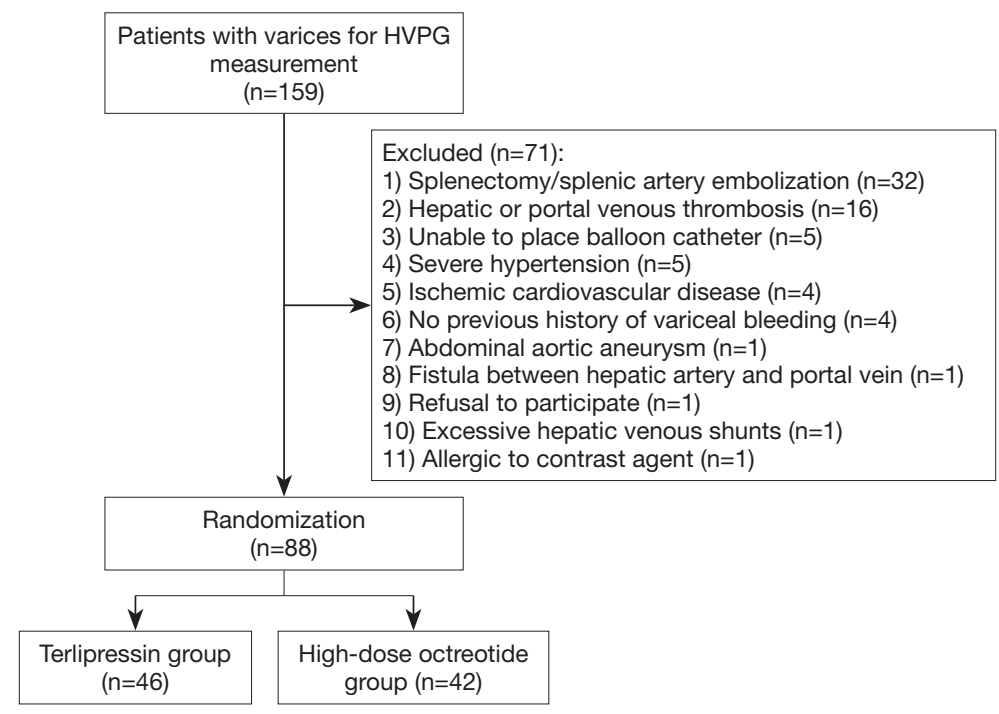

Figure 1 Flow diagram of the study process and subject selection.

administered through the peripheral vein. The HVPG was measured according to the study protocol. The puncture site was then pressed for 15-20 min under close observation after the procedure.

\section{Study protocol}

The random table method was used for randomization, and sealed opaque envelopes were used for the allocation. Baseline HVPG, heart rate (HR), and mean arterial pressure (MAP) measurements of patients who met the inclusion criteria were recorded before vasoactive drug administration. MAP $=$ diastolic blood pressure + (systolic blood pressure - diastolic blood pressure)/3 (12). The vasoactive drugs used in the study were terlipressin (Hybio, Shenzhen, China) and octreotide (Novartis, Basel, Switzerland). After baseline measurements, patients were randomly assigned into two groups: the terlipressin group $(n=46)$ and the high-dose octreotide group $(n=42)$. The patients in the terlipressin group received an intravenous injection of $2 \mathrm{mg}$ terlipressin, while those in the high-dose octreotide group received an initial intravenous injection of $100 \mu \mathrm{g}$ octreotide followed by continuous intravenous infusion of $50 \mu \mathrm{g} / \mathrm{h}$. HVPG, HR, and MAP were originally recorded at 10,20 min after the start of drug administration. Since the differences in HVPG between the two groups were not obvious until $20 \mathrm{~min}$ and no further reduction in HVPG was found after $30 \mathrm{~min}$, we decided to prolong the observation time to $30 \mathrm{~min}$. The patients were observed for adverse drug events for 24 hours. The primary endpoint was the change in HVPG. The secondary endpoints were the changes in HR and MAP, hemodynamic responses, and the incidence of adverse events. Hemodynamic responses were defined as HVPG having decreased more than $20 \%$ from baseline or below $12 \mathrm{mmHg}$ after drug administration at the time point (7).

\section{Statistical analysis}

All analyses were performed using SPSS v20.0 (SPSS Inc., Chicago, IL, USA). The sample size estimation was performed by assuming that the standard deviation of HVPG ranged between $5 \%$ and $15 \%$, and the mean difference of HVPG ranged from $10 \%$ to $20 \%$. To obtain an $\alpha$-error of 0.05 and a power of $0.8,40$ patients in each group were sufficient. Categorical data were compared using the $\chi^{2}$ test, while continuous data were compared using the Student's $t$-test or the Mann-Whitney ranksum test. All tests were two-sided, and $\mathrm{P}$ values $<0.05$ were considered statistically significant.

\section{Results}

Between February 1, 2014, and January 31, 2016 a total of 88 patients were included in the study. After randomization, 46 patients were assigned to the terlipressin group and 42 to the high-dose octreotide group (Figure 1). The clinical characteristics of the patients in the two groups are shown 
Table 1 Baseline characteristics

\begin{tabular}{|c|c|c|c|}
\hline Characteristics & Terlipressin group $(n=46)$ & High-dose octreotide group ( $n=42)$ & $P$ value \\
\hline Age (year) & $53 \pm 10$ & $51 \pm 13$ & 0.381 \\
\hline Etiology (HBV/HCV/alcoholic/others), n (\%) & $\begin{array}{c}24(52.2 \%) / 1(0.02 \%) / 8 \\
(17.4 \%) / 13(28.3 \%)\end{array}$ & $23(54.8 \%) / 1(0.02 \%) / 4(9.5 \%) / 14(33.3 \%)$ & 0.546 \\
\hline Albumin $(g / L)$ & $32.4 \pm 4.6$ & $33.4 \pm 6.2$ & 0.368 \\
\hline Total bilirubin ( $\mu \mathrm{mol} / \mathrm{L})$ & $21.0 \pm 9.1$ & $21.7 \pm 11.2$ & 0744 \\
\hline Creatinine $(\mu \mathrm{mol} / \mathrm{L})$ & $67.4 \pm 21.6$ & $68.9 \pm 12.2$ & 0.791 \\
\hline Child-Pugh class $(\mathrm{A} / \mathrm{B} / \mathrm{C})$ & $19 / 24 / 3$ & $22 / 19 / 1$ & 0.444 \\
\hline Heart rate (beats/min) & $77.6 \pm 12.5$ & $78.2 \pm 15.7$ & 0.842 \\
\hline Mean arterial pressure $(\mathrm{mmHg})$ & $90.4 \pm 12.3$ & $95.4 \pm 13.0$ & 0.077 \\
\hline WHVP $(\mathrm{mmHg})$ & $24.0 \pm 6.4$ & $23.8 \pm 6.5$ & 0.885 \\
\hline FHVP (mmHg) & $7.7 \pm 4.4$ & $6.4 \pm 3.4$ & 0.132 \\
\hline HVPG $(\mathrm{mmHg})$ & $16.3 \pm 6.4$ & $17.4 \pm 6.6$ & 0.433 \\
\hline$\Delta \mathrm{HVPG}_{10 \min }(\mathrm{mmHg})$ & $1.6 \pm 2.7$ & $2.2 \pm 3.6$ & 0.366 \\
\hline
\end{tabular}

HVPG, hepatic venous pressure gradient; FHVP, free hepatic venous pressure; WHVP, wedged hepatic venous pressure; HBV, hepatitis B; HCV, hepatitis C.

in Table 1.

\section{Changes in portal hemodynamics}

The HVPG of the terlipressin group decreased significantly at $10 \min (14.7 \pm 5.9$ vs. $16.3 \pm 6.4, \mathrm{P}<0.001), 20 \min (14.0 \pm 6.1$ vs. $16.3 \pm 6.4, \mathrm{P}<0.001)$, and $30 \mathrm{~min}(13.8 \pm 6.1$ vs. $16.3 \pm 6.4$, $\mathrm{P}<0.001)$ after drug administration compared to the baseline HVPG. Furthermore, the HVPG was significantly decreased at $20 \mathrm{~min}$ compared to $10 \mathrm{~min}(\mathrm{P}=0.038)$ and at $30 \mathrm{~min}$ compared to $20 \mathrm{~min}(\mathrm{P}=0.028)$, indicating that terlipressin resulted in a persistent reduction of HVPG without rebounding. The HVPG in the high-dose octreotide group also dropped significantly from baseline at $10 \min (15.1 \pm 5.8$ vs. $17.4 \pm 6.6, \mathrm{P}<0.001), 20 \min (15.3 \pm 6.2$ vs. $17.4 \pm 6.6, \mathrm{P}=0.001)$, and $30 \mathrm{~min}(16.1 \pm 6.0$ vs. $17.4 \pm 6.6$,
$\mathrm{P}<0.001)$. However, the HVPG tended to rise after $10 \mathrm{~min}$, indicating that the high-dose octreotide effect might be relatively transient (Figure 2). The $\triangle \mathrm{HVPG}$ was defined as the difference between the HVPG before and after drug administration, and $\triangle \mathrm{HVPG}_{10 \text { min }}, \Delta \mathrm{HVPG}_{20 \text { min }}$, and $\triangle \mathrm{HVPG}_{30 \text { min }}$ were not significantly different between the two groups (Table 1).

\section{Changes in systemic bemodynamics}

The HR of the terlipressin group significantly decreased at $10 \mathrm{~min}(69.9 \pm 11.3$ vs. $77.6 \pm 12.5, \mathrm{P}=0.003), 20 \mathrm{~min}$ $(66.9 \pm 11.2$ vs. $77.6 \pm 12.5, \mathrm{P}<0.001)$, and $30 \mathrm{~min}(69.5 \pm 12.0$ vs. 77.6 $\pm 12.5, \mathrm{P}=0.004)$ compared to baseline. The HR of the high-dose octreotide group was not significantly different at $10 \mathrm{~min}(76.8 \pm 14.2$ vs. $78.2 \pm 15.7, \mathrm{P}=0.718)$, 


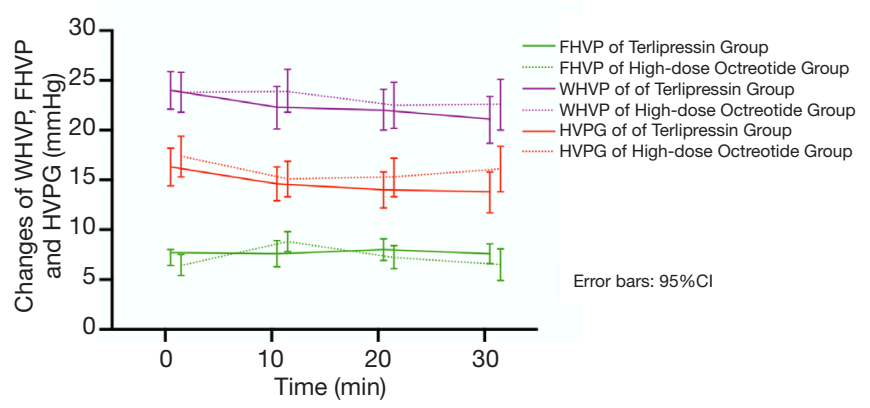

Figure 2 The changes in HVPG, FHVP, and WHVP at 10, 20, and $30 \mathrm{~min}$ after drug administration. HVPG, hepatic venous pressure gradient; FHVP, free hepatic venous pressure; WHVP, wedged hepatic venous pressure.
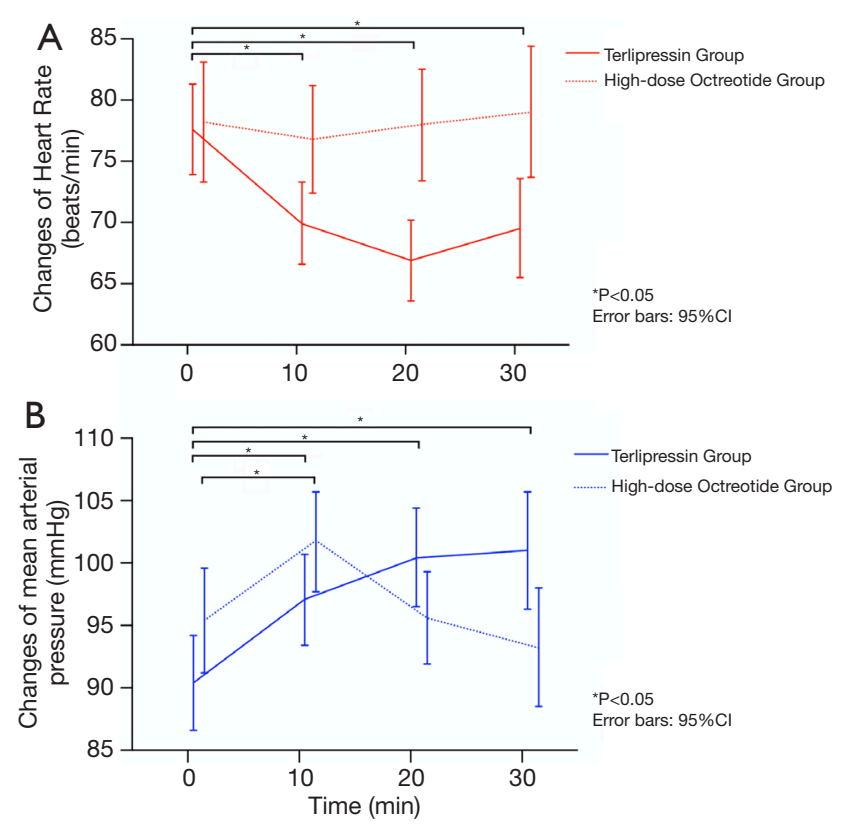

Figure 3 The changes in (A) heart rate and (B) mean arterial pressure at 10,20, and $30 \mathrm{~min}$ after drug administration.

$20 \mathrm{~min}(78.0 \pm 14.6$ vs. $78.2 \pm 15.7, \mathrm{P}=0.982)$, and $30 \mathrm{~min}$ (79.0 \pm 14.1 vs. $78.2 \pm 15.7, \mathrm{P}=0.793)$. The HR of the terlipressin group was significantly lower than the highdose octreotide group at $10 \min (69.9 \pm 11.3$ vs. $76.8 \pm 14.2$, $\mathrm{P}=0.014), 20 \min (66.9 \pm 11.2$ vs. $78.0 \pm 14.6, \mathrm{P}<0.001)$, and 30 min $(69.5 \pm 12.0$ vs. $79.0 \pm 14.1, \mathrm{P}=0.005)$ (Figure $3 A)$. Compared to baseline, the MAP was significantly elevated at $10 \mathrm{~min}(97.1 \pm 11.5$ vs. $90.4 \pm 12.3, \mathrm{P}=0.012)$, $20 \mathrm{~min}(100.4 \pm 12.4$ vs. $90.4 \pm 12.3, \mathrm{P}<0.001)$, and $30 \mathrm{~min}$
$(101.0 \pm 13.1$ vs. $90.4 \pm 12.3, \mathrm{P}=0.001)$ after terlipressin administration. Similar changes in the MAP were found at $10 \mathrm{~min}(101.8 \pm 12.5$ vs. $95.4 \pm 13.0, \mathrm{P}=0.03)$ in the highdose octreotide group, but not at $20 \mathrm{~min}(95.6 \pm 11.7 \mathrm{vs}$. $95.4 \pm 13.0, \mathrm{P}=0.768)$ and $30 \mathrm{~min}(93.2 \pm 12.1$ vs. $95.4 \pm 13.0$, $\mathrm{P}=0.425)$. The MAP was not significantly different between the terlipressin group and the high-dose octreotide group at $10 \mathrm{~min}(97.1 \pm 11.5$ vs. $101.8 \pm 12.5, \mathrm{P}=0.08)$ or $20 \mathrm{~min}$ (100.4 \pm 12.4 vs. $95.6 \pm 11.7, \mathrm{P}=0.076)$, though the difference was significant at $30 \mathrm{~min}(101.0 \pm 13.2$ vs. $93.2 \pm 12.1$, $\mathrm{P}=0.021$ ) (Figure $3 B$ ). These results indicated that terlipressin, but not high-dose octreotide, led to decreased $\mathrm{HR}$ and an increase in MAP.

\section{Hemodynamic response}

The overall response rates increased over time in both groups (Table 2). The overall response rates were not significantly different between the two groups at $10 \mathrm{~min}$ (29.5\% vs. $38.1 \%, \mathrm{P}=0.402), 20 \mathrm{~min}(33.3 \%$ vs. $38.1 \%$, $\mathrm{P}=0.642)$, and $30 \mathrm{~min}(52.8 \%$ vs. $44.8 \%, \mathrm{P}=0.524)$. The response rates were further adjusted for factors such as gender (male vs. female), Child-Pugh grading (A vs. B/C), baseline HVPG ( $\leq 12$ vs. $>12 \mathrm{mmHg})$, and etiology of liver cirrhosis (HBV vs. alcoholic vs. others). The terlipressin group tended to have relatively higher response rates at 30 min after drug administration compared to the highdose octreotide group in those with Child-Pugh B/C (46.3\% vs. $39.1 \%, \mathrm{P}=0.127)$ or baseline $\mathrm{HVPG}>12 \mathrm{mmHg}(85.2 \%$ vs. $62.5 \%, \mathrm{P}=0.064)$. The response rate at $30 \mathrm{~min}$ was significantly higher in the terlipressin group than the highdose octreotide group in those with alcoholic liver cirrhosis [6/6 (100\%) vs. 0/4 (0\%), $\mathrm{P}=0.005]$.

\section{Adverse drug events}

The most commonly documented adverse events of the vasoactive drugs were abdominal pain, nausea, vomiting, diarrhea, and headache. Rare adverse events included bradycardia, dizziness, flushing, myocardial ischemia, and acute mesenteric ischemia. Overall, the adverse drug events occurred in $6 / 88(0.1 \%)$ patients and were transient. There were no serious adverse events such as myocardial ischemia. No significant differences in the incidence rates of adverse events were observed between the terlipressin and the highdose octreotide groups (Table 3). 
Table 2 Response rates between the two groups

\begin{tabular}{|c|c|c|c|}
\hline The time point after drug administration & Terlipressin group $(n=46)$ & High-dose octreotide group ( $n=42)$ & $P$ value \\
\hline $20 \mathrm{~min}$ & $15 / 45(33.3 \%)$ & $16 / 42(38.1 \%)$ & 0.642 \\
\hline $30 \mathrm{~min}$ & $19 / 36(52.8 \%)$ & $13 / 29(44.8 \%)$ & 0.524 \\
\hline
\end{tabular}

Table 3 Adverse drug events in the two groups

\begin{tabular}{|c|c|c|c|}
\hline Adverse drug events & Terlipressin group $(n=46)$ & High-dose octreotide group $(n=42)$ & $P$ value \\
\hline Nausea or vomiting & $1(2.2 \%)$ & $2(4.8 \%)$ & 0.504 \\
\hline Myocardial ischemia & $0(0 \%)$ & $0(0 \%)$ & NA \\
\hline Arrhythmia & $0(0 \%)$ & $1(2.4 \%)$ & 0.293 \\
\hline Total & $2(4.3 \%)$ & $4(9.5 \%)$ & 0.336 \\
\hline
\end{tabular}

NA, not applicable.

\section{Discussion}

The Baveno VI consensus recommended that vasoactive drugs such as somatostatin, terlipressin, or octreotide should be used as soon as possible once acute variceal bleeding occurs (1). It is important to maintain hemodynamic stability before patients get access to endoscopy. Therefore, it is necessary to study the immediate hemodynamic effects of different vasoactive drugs to determine the optimal firstline strategy.

A previous study suggested using terlipressin to control variceal bleeding as it was proven to be the only vasoactive drug that improved survival (13). However, this advantage over somatostatin was not significant when it was used as an adjuvant to endoscopy treatments (14). On the other hand, octreotide's survival superiority over alternative vasoactive drugs was not confirmed in a meta-analysis (2). A more recent meta-analysis demonstrated that there was no difference in 7-day mortality among the vasoactive drugs (3). Further treatments, including endoscopy and TIPS, often covered the true efficacy of initial vasoactive drugs. Thus, instead of the long-term clinical endpoints (rebleeding, survival, etc.), we focused on the immediate hemodynamic effects of terlipressin and high-dose octreotide on HVPG.

Our study results were consistent with previous research, which found that terlipressin significantly reduced HVPG, decreased HR, and increased MAP (15). Although there was no significant difference between terlipressin and highdose octreotide in terms of reducing HVPG within 30 min, the effects of terlipressin lasted longer without a rebound. On the other hand, Baik et al. (10) showed that HVPG was decreased to the lowest level at $1 \mathrm{~min}$ after octreotide administration but returned to the baseline level after $5 \mathrm{~min}$. Villanueva et al. (9) found that the hemodynamic changes induced by high-dose somatostatin were more consistent than its regular dose. Therefore, it remained in question whether octreotide followed the same pattern. Regulardose octreotide was administered as a $100 \mu \mathrm{g}$ intravenous bolus dose followed by $25 \mu \mathrm{g} / \mathrm{h}$ continuous infusion $(16,17)$. Our study's high-dose octreotide was administered as $100 \mu \mathrm{g}$ intravenous bolus followed by $50 \mu \mathrm{g} / \mathrm{h}$ continuous infusion. Our results confirmed a rise in HVPG after $10 \mathrm{~min}$ in the high-dose octreotide group. However, HVPG was significantly reduced compared to baseline at 10, 20, and $30 \mathrm{~min}$ after administration. This indicated that higher doses had more persistent hemodynamic effects. It is worth noting that octreotide blunted postprandial increase in portal venous pressure by inhibiting vasodilatory peptide release. Such effects were related to drug doses (18). This could blunt sudden peaks of portal venous pressure after meals and potentially prevent variceal rupture.

It was suggested that the rebound of HVPG when using octreotide was due to the rapid desensitization of the somatostatin receptors (19). Furthermore, the long- 
acting octreotide had no significant hemodynamic benefits in treating portal hypertension (20). Recent studies demonstrated that the continuous infusion of terlipressin stably reduced portal pressure with less HR and MAP fluctuations than traditional bolus infusion and prevented renal impairment $(21,22)$.

This study had several limitations. Firstly, blinding was inapplicable in the study. However, objective parameters such as HVPG, HR, and MAP were adopted as endpoints to avoid information bias. Another limitation was that the patients included in our study did not have active variceal bleeding out of ethical considerations. The baseline HVPG level was often not available because vasoactive drugs were administered immediately once variceal bleeding was suspected. Lastly, the response rate at $30 \mathrm{~min}$ was significantly higher in the terlipressin group with alcoholic liver cirrhosis. These data should be interpreted with caution since the sample size was relatively small. Thus, future studies with larger sample sizes are required to verify the conclusions.

\section{Conclusions}

In summary, both terlipressin and high-dose octreotide were effective and safe in reducing HVPG. The pharmacodynamic effect of terlipressin persisted longer. The terlipressin group had higher response rates in those with alcoholic cirrhosis.

\section{Acknowledgments}

Funding: This study was partially supported by the Innovation Fund of Shanghai Scientific Committee (No. 15411950501).

\section{Footnote}

Reporting Checklist: The authors have completed the CONSORT reporting checklist. Available at http://dx.doi. org/10.21037/atm-20-6774

Data Sharing Statement: The data of the study will be available on reasonable request. Available at http://dx.doi. org/10.21037/atm-20-6774

Peer Review File: Available at http://dx.doi.org/10.21037/ atm-20-6774
Conflicts of Interest: All authors have completed the ICMJE uniform disclosure form (available at http://dx.doi. org/10.21037/atm-20-6774). The authors have no conflicts of interest to declare.

Ethical Statement: The authors are accountable for all aspects of the work in ensuring that questions related to the accuracy or integrity of any part of the work are appropriately investigated and resolved. The study was conducted following the Declaration of Helsinki (as revised in 2013). The ethics board approved the study of Zhongshan Hospital and Shandong Provincial Hospital (No. B2014-025), and informed consent was obtained from all individual participants.

Open Access Statement: This is an Open Access article distributed in accordance with the Creative Commons Attribution-NonCommercial-NoDerivs 4.0 International License (CC BY-NC-ND 4.0), which permits the noncommercial replication and distribution of the article with the strict proviso that no changes or edits are made and the original work is properly cited (including links to both the formal publication through the relevant DOI and the license). See: https://creativecommons.org/licenses/by-nc-nd/4.0/.

\section{References}

1. de Franchis R. Expanding consensus in portal hypertension: report of the Baveno VI Consensus Workshop:Stratifying risk and individualizing care for portal hypertension. J Hepatol 2015;63:743-52.

2. Corley DA, Cello JP, Adkisson W, et al. Octreotide for acute esophageal variceal bleeding: a meta-analysis. Gastroenterology 2001;120:946-54.

3. Wells M, Chande N, Adams P, et al. Metaanalysis:vasoactive medications for the management of acute variceal bleeds. Aliment Pharmacol Ther 2012;35:1267-78.

4. Seo YS, Park SY, Kim MY, et al. Lack of Difference Among Terlipressin, Somatostatin, and Octreotide in the Control of Acute Gastroesophageal Variceal Hemorrhage. Hepatology 2014;60:954-63.

5. Wang C, Han J, Xiao L, et al. Efficacy of vasopressin/ terlipressin and somatostatin/octreotide for the prevention of early variceal rebleeding after the initial control of bleeding: a systematic review and meta-analysis. Hepatol Int 2015;9:120-9. 


\section{Page 8 of 8}

6. Merkel C, Montagnese S. Hepatic venous pressure gradient measurement in clinical hepatology. Dig Liver Dis 2011;43:762-7.

7. D'Amico G, Garcia-Pagan JC, Luca A, et al. Hepatic Vein Pressure Gradient Reduction and Prevention of Variceal Bleeding in Cirrhosis: a Systematic Review. Gastroenterology 2006;131:1611-24.

8. Villanueva C, Ortiz J, Miñana J, et al. Somatostatin Treatment and Risk Stratification by Continuous Portal Pressure Monitoring During Acute Variceal Bleeding. Gastroenterology 2001;121:110-7.

9. Villanueva C, Planella M, Aracil C, et al. Hemodynamic Effects of Terlipressin and High Somatostatin Dose during Acute Variceal Bleeding in Nonresponders to the Usual Somatostatin Dose. Am J Gastroenterol 2005;100:624-30.

10. Baik SK, Jeong PH, Ji SW, et al. Acute Hemodynamic Effects of Octreotide and Terlipressin in Patients with Cirrhosis: a Randomized Comparison. Am J Gastroenterol 2005;100:631-5.

11. Cirera I, Feu F, Luca A, et al. Effects of bolus injections and continuous infusions of somatostatin and placebo in patients with cirrhosis: a double-blind hemodynamic investigation. Hepatology 1995;22:106-11.

12. Kalambokis G, Economou M, Paraskevi K, et al. Effects of somatostatin, terlipressin and somatostatin plus terlipressin on portal and systemic hemodynamics and renal sodium excretion in patients with cirrhosis. J Gastroenterol Hepatol 2005;20:1075-81.

13. Ioannou GN, Doust J, Rockey DC. Systematic review: terlipressin in acute oesophageal variceal haemorrhage. Aliment Pharmacol Ther 2003;17:53-64.

14. Hung TH, Tsai CC, TsenK GC, et al. No mortality difference following treatment with terlipressin or somatostatin in cirrhotic patients with gastric variceal hemorrhage. Saudi J Gastroenterol 2016;22:220-25.

Cite this article as: Li B, Chen J, Zhang CQ, Wang GC, Hu JH, Luo JJ, Zhang W, Wei YC, Zeng XQ, Chen SY. The pharmacodynamic effect of terlipressin versus high-dose octreotide in reducing hepatic venous pressure gradient: a randomized controlled trial. Ann Transl Med 2021;9(9):793. doi: 10.21037/atm-20-6774
Li et al. Terlipressin vs. high-dose octreotide in reducing HVPG

15. Narahara Y, Kanazawa H, Taki Y, et al. Effects of terlipressin on systemic, hepatic and renal hemodynamics in patients with cirrhosis. J Gastroenterol Hepatol 2009;24:1791-7.

16. Hwang SJ, Lin HC, Chang CF, et al. A randomized controlled trial comparing octreotide and vasopressin in the control of acute esophageal variceal bleeding. J Hepatol 1992;16:320-5.

17. Pedretti G, Elia G, Calzetti C, et al. Octreotide versus terlipressin in acute variceal hemorrhage in liver cirrhosis. Emergency control and prevention of early rebleeding. Clin Investig 1994;72:653-9.

18. Vorobioff JD, Gamen M, Kravetz D, et al. Effects of Long-term Propranolol and Octreotide on Postprandial Hemodynamics in Cirrhosis: a Randomized, Controlled Trial. Gastroenterology 2002;122:916-22.

19. Escorsell A, Bandi JC, Andreu V, et al. Desensitization to the effects of intravenous octreotide in cirrhotic patients with portal hypertension. Gastroenterology 2001;120:161-9.

20. Chandok N, Kamath PS, Blei A, et al. Randomised clinical trial: the safety and efficacy of long-acting octreotide in patients with portal hypertension. Aliment Pharmacol Ther 2012;35:904-12.

21. 21 Ding C, Wu XJ, Fan XX, et al. Hemodynamic effects of continuous versus bolus infusion of terlipressin for portal hypertension: a randomized comparison. J Gastroenterol Hepatol 2013;28:1242-6.

22. Zhang J, Liu J, Wu Y, et al. Effect of terlipressin on renal function in cirrhotic patients with acute upper gastrointestinal bleeding. Ann Transl Med 2020;8:340.

(English Language Editors: C. Betlazar-Maseh and J. Chapnick) 